\title{
Progress on a Be Cavity Design
}

\author{
D. Li ${ }^{\mathrm{a}}$, R. Palmer ${ }^{\mathrm{b}}$, D. Stratakis ${ }^{\mathrm{b}}$, S. Virostek ${ }^{\mathrm{a}}$, and M. S. Zisman ${ }^{\mathrm{a}}$ \\ ${ }^{a}$ Lawrence Berkeley National Laboratory, Berkeley, CA 94720 U.S.A. \\ ${ }^{b}$ Brookhaven National Laboratory, Upton, NY 11973 U.S.A.
}

\begin{abstract}
Previous RF experiments with normal-conducting cavities have demonstrated that there is a significant degradation in maximum gradient when the cavity is subjected to a strong axial magnetic field. We have developed a model suggesting that a cavity with beryllium walls may perform better than copper cavities. In this paper we outline the issues that led us to propose fabricating a Be-wall cavity. We also discuss a concept for fabricating such a cavity and mention some of the manufacturing issues we expect to face.
\end{abstract}

Keywords: Normal-conducting RF; RF breakdown; Be cavity design PACS: 29.20.-c; 29.20.Ej

\section{INTRODUCTION}

In the past few years, MuCool RF studies [1] have shown that there is a significant reduction in maximum gradient in normal-conducting cavities immersed in a strong solenoidal field. Unfortunately, this is precisely the configuration needed for the cooling channel of a Neutrino Factory or a Muon Collider.

A number of tests have been made with $805-\mathrm{MHz}$ cavities. For "vacuum" cavities with Be windows the degradation is more than a factor of two, as shown in Fig. 1. Tests at $201 \mathrm{MHz}$ show a qualitatively similar effect, though less marked. However, these latter tests suffer from lack of a large-diameter solenoid coil. Such a coil is presently being fabricated in China [2] and should be available for use in early 2012 .

One caveat to the present results is that we observed breakdown problems in the coupler region in both cavities. This effect needs to be eliminated before we draw any final conclusions from our previous test results. Modifications to our $805-\mathrm{MHz}$ cavity to improve the coupler have been completed and the cavity will be retested shortly.

Some breakdown models have suggested that Be may be a better wall material than $\mathrm{Cu}$, and this has motivated us to undertake the design and fabrication of an $805-\mathrm{MHz}$ Be-wall cavity. When ready, it will be tested in the MuCool Test Area (MTA) at Fermilab.

\section{MODEL}

The damage and breakdown observed in 201- and 805$\mathrm{MHz}$ cavities when operated in magnetic fields may be

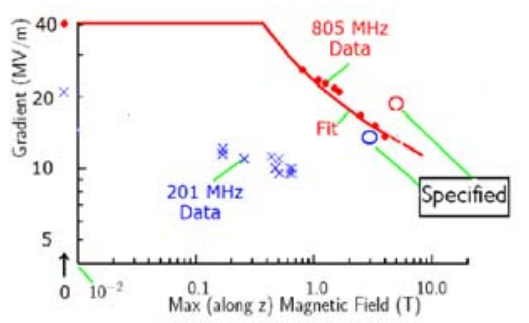

FIGURE 1. Maximum gradient achieved in MTA tests vs. axial magnetic field strength. At both frequencies we fall short of the desired gradient for a muon cooling channel.

caused by the impact of field-emitted electrons focused by the magnetic fields onto a small area of the copper surfaces in the cavity, as illustrated in Fig. 2(a) [3]. Resultant surface damage would then be due to fatigue [4] from cyclical strains induced by local heating from the electrons. A systematic study to investigate fatigue from pulsed RF heating on copper was performed at SLAC. Data were obtained [5] using a special $11.4-\mathrm{GHz}$ cavity with no surface electric fields but with surface currents induced by the RF magnetic field (Fig. 2(b)). Damage on soft copper
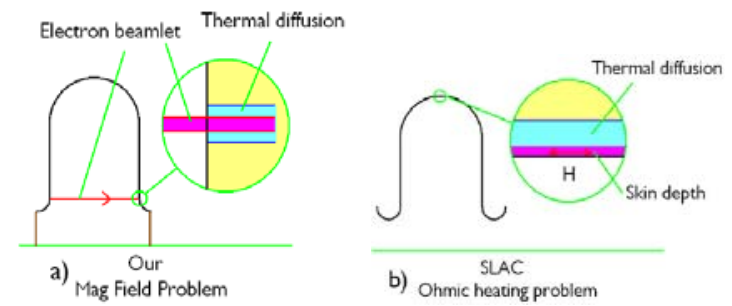

FIGURE 2. (a) assumed heating from dark-current beamlet hitting opposite wall of the cavity; (b) corresponding surface current heating mechanism seen in the SLAC experiments. 
was observed after cyclical heating with a temperature rise of only $\sim 45^{\circ} \mathrm{C}$. We assume here that a similar effect will occur due to "beamlet" heating that results in this temperature rise.

To mitigate such fatigue damage, we seek materials that have the following desirable properties:

- low coefficient of thermal expansion

- high specific heat

- high thermal conductivity

- (for surface heating) high electrical conductivity

Materials considered include copper, beryllium, and aluminum. The choice of beryllium was based on its low density, low thermal expansion, and the observation that Be windows employed in the earlier RF tests suffered no visible damage from the breakdown events.

\section{Estimation of Strain}

Our approach was to use approximate calculations to estimate the temperature rise and the resultant strain. The equations employed were:

$$
\begin{aligned}
& \Delta T \propto \frac{d E}{d x} \int_{0}^{t} \frac{Q(T)}{A_{\text {beam }} \rho C_{p}(T)} d T \\
& \text { Strain } \propto \frac{d E}{d x} \int_{0}^{t} \frac{Q(T) \alpha(T)}{A_{\text {beam }} \rho C_{p}(T)} d T
\end{aligned}
$$

where $\alpha(T)$ is the coefficient of thermal expansion, $A_{\text {beam }}$ is the beamlet area at the wall surface, and $Q(T)$ accounts for thermal diffusion, which increases the transverse size of the heat zone.

To estimate thermal diffusion, we assumed a diffusion size comparable to the spot size, in which case $Q(T)=d(293) / d(T)$, where

$$
d(T)=\sqrt{\frac{k(T) \tau}{\rho C_{p}(T)}}
$$

$k(T)$ is thermal conductivity and $\tau$ is cavity decay time.

We normalized results from Eq. (1) to give a $\Delta T$ of $45^{\circ} \mathrm{C}$ for room temperature copper-the value that resulted in damage in the SLAC experiments. Results for all three materials, Fig. 3; show Be gives the lowest $\Delta T$.

The result of using Eq. (2) to estimate the strain is shown in Fig. 4. We see that Be has much less strain than $\mathrm{Cu}$, whereas $\mathrm{Al}$ is not very different than $\mathrm{Cu}$. For case $b$, where thermal diffusion matters, we predict a marked improvement for Be at low temperatures.

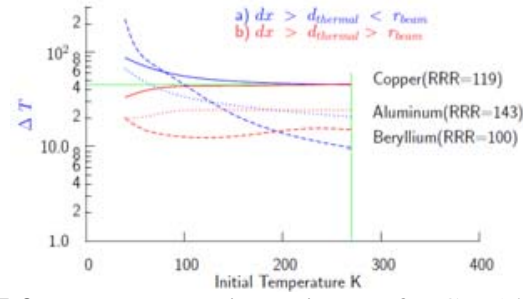

FIGURE 3. Temperature rise estimates for $\mathrm{Cu}, \mathrm{Al}$, and $\mathrm{Be}$.

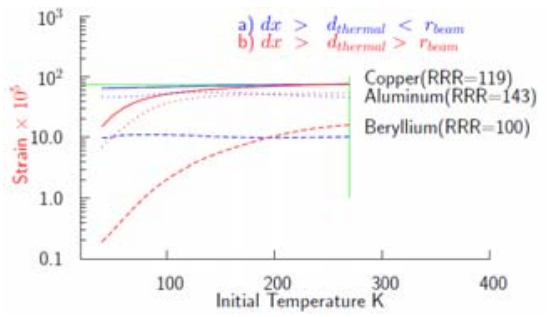

FIGURE 4. Strain estimates for $\mathrm{Cu}, \mathrm{Al}$, and $\mathrm{Be}$.

\section{Estimated Damage Thresholds}

Finally, in Fig. 5 we estimate breakdown gradients for the three materials, assuming that damage occurs at the same strain as for $\mathrm{Cu}$ at $\Delta T=45^{\circ} \mathrm{C}$. We see again that Be looks better than the other materials, and that the shape of the curves is roughly consistent with what has been observed experimentally. This motivates us to build such a cavity and test it.

\section{Conclusions from Modeling}

From these results, it appears that beryllium is a very promising cavity material and that it may solve, or at least mitigate, the gradient degradation found for copper cavities in an axial magnetic field. Aluminum may also be somewhat better than copper, but would require an anti-multipactor coating such as TiN to be usable in this application. The main drawbacks of beryllium are that it is a difficult material to work with and is expensive. This aspect will be discussed below.

Before embarking on cavity fabrication, we plan a test program using back-to-back Be "buttons" in our existing pillbox cavity. The buttons will have a field enhancement factor of three to ensure that breakdown occurs at the desired location.

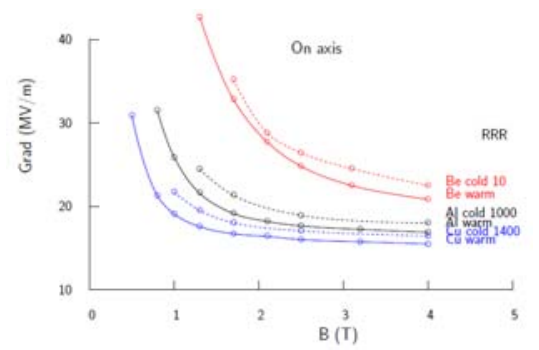

FIGURE 5. Damage threshold estimates for $\mathrm{Cu}, \mathrm{Al}$, and $\mathrm{Be}$. 


\section{CAVITY DESIGN AND FABRICATION}

A cavity concept has been developed that includes two bolted halves connected with both vacuum and RF seals. This choice offers accessibility advantages over an e-beam-welded design. There are two options for the body, solid copper or copper-plated Hastelloy. The coupling port will be slotted in the side wall to allow the cavity to fit in the bore of an existing solenoid magnet in the MTA. A schematic of the proposed cavity illustrating the spatial constraints is shown in Fig. 6, and an initial CAD model is shown in Fig. 7.

Several options for the side walls are being considered, including

- thin $(0.5 \mathrm{~mm})$ Be foil brazed to side walls

- thick (6 mm) Be plates brazed to side walls

- solid Be walls (no brazing)

An all-Be cavity is deemed to be impractical and is not under consideration.

Based on the initial CAD model, the next steps are to evaluate the cost of the Be material, to compare the costs of the various fabrication options, and to analyze the manufacturing risks (brazing, bolted joints, and machining processes, especially for the coupling port).

\section{Manufacturing Issues}

There are a number of issues with manufacture of the proposed cavity, most of which are related to handling beryllium. For example, only a few select shops are available to do Be machining, and the same is true for brazing operations.

If a solid copper body is used, differential expansion of the dissimilar materials must be accounted for. Hastelloy has a coefficient of thermal expansion similar to Be, but its brazing properties need to be assessed. The transition from Be side walls to the rounded corner of the cavity at its inner diameter is likely to present some challenges. In addition, the brazed interface between the Be cavity wall and the cavity body at the coupling port is tricky and must be handled carefully. Finally, the joint between the two cavity halves must be designed to accommodate a good RF seal as well as a good vacuum seal.

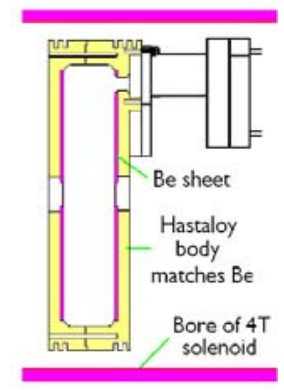

FIGURE 6. Schematic of Be cavity.

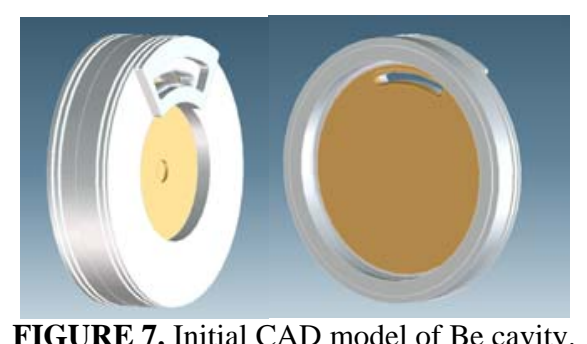

There are a number of remaining tasks to produce the cavity. After completing the conceptual design, mechanical analysis and trade-off studies must be carried out. These will lead to a final conceptual design, after which we will prepare the fabrication drawings and procure the long-lead materials. Finally, the cavity components will be fabricated and then brazed, coated, and assembled.

\section{SUMMARY}

We have developed a model of cavity damage based on cyclic heating of the cavity walls by tightly focused dark-current beamlets. With a few simplifying assumptions, damage thresholds have been estimated for several materials. Beryllium appears to be the most promising, and we are designing a Be-wall cavity to test this. Beryllium button tests will first be performed to validate the model. If these are successful, the cavity will be built and tested at the Fermilab MTA.

\section{ACKNOWLEDGMENTS}

We thank our MAP colleagues for help with the RF test program and for many informative discussions.

Work supported by the Office of Science, U.S. Dept. of Energy Contract Nos. DE-AC02-05CH11231 (LBNL) and DE-AC02-98CH10886 (BNL).

\section{REFERENCES}

1. D. Huang et al., in Proc. 2009 Particle Accelerator Conf., Vancouver, BC, Canada, May 4-8, 2009, p. 888; see http://accelconf.web.cern.ch/AccelConf/PAC2009/papers /tu5pfp032.pdf.

2. L. Wang et al., in Proc. 2009 Particle Accelerator Conf., Vancouver, BC, Canada, May 4-8, 2009, p. 289; http://accelconf.web.cern.ch/AccelConf/PAC2009/papers /mo6pfp069.pdf.

3. R. B. Palmer et al., Phys. Rev. Spec. Topics AB $\underline{12}$, 031002 (2009).

4. D. Stratakis, J. C. Gallardo, and R. B. Palmer, Nucl. Instr. Meth. A 620, 147 (2010).

5. D. Pritzkau and R. H. Siemann, Phys. Rev. Spec. Topics AB $\underline{5}, 112002$ (2002). 